\title{
Eversion endarterectomy of the external iliac artery
}

\author{
Anna Barczak ${ }^{1,2}$, Sergiusz Iljin ${ }^{1,2}$, Konrad Koszczyk², Jaroslaw Miszczuk ${ }^{1,2}$ \\ 'Vascular Surgery Clinic, Voivodeship Polyclinic Hospital in Kielce, Poland \\ ${ }^{2}$ General and Vascular Surgery Department, Voivodeship Specialistic Hospital in Czestochowa, Poland
}

\begin{abstract}
Introduction. To redraw attention to the meaning and value of eversion endarterectomy (EA) of external iliac arteries as a still effective surgical method used in the treatment of advanced arterial occlusive disease. The authors of this article would like to present their experience in this scope.
\end{abstract}

Material and methods. This is a presentation of the applied method of treatment; it comprises the descriptions of nine consecutive cases of patients suffering from atherosclerotic stenosis or occlusion of external iliac arteries and advanced lower limb ischaemia, manifesting in a short distance of claudication, rest pain and commencing necrotic lesions in the lower limbs, who were operated on in the years 2013-20/4, for whom the restoration of patency in the external iliac arteries was achieved by use of eversion endarterectomy. It also comprises two other patients for whom the restoration of patency in the external iliac arteries was achieved by eversion endarterectomy but who were operated on for acute lower limb ischaemia.

Results. In all but one patient the restoration of patency by eversion endarterectomy was successful, resulted in improvement of blood flow in the limb and reduction of the patients' ailments. In one case there was no improvement and the patient required more surgical interventions. In the same case a retroperitoneal aneurysm arose, requiring surgical removal. In another case early thrombosis of the operated artery developed and was successfully treated with thrombectomy. The other patients reported alleviation or absence of pain directly after the surgery. In our patients' group we noted one serious undesired extra-vascular event — a myocardial infarction type 2. Mortality after 30 days was zero.

Conclusions. Eversion endarterectomy of the external iliac artery is an effective and relatively simple surgical method of known and proven effectiveness. It constitutes a bit forgotten but valuable supplement to an armamentarium of surgical methods in the treatment of atherosclerosis of an aortoiliac segment.

Key words: endarterectomy, external iliac artery, arterial occlusive disease

Acta Angiol 2015; 21, 2: 40-46

\section{Introduction}

The history of endarterectomy begins in 1946 from the successful endarterectomy of the common femoral artery performed by the Portuguese surgeon, Joao Cid dos Santos, at the University of Lisbon. The earlier attempts had ended with failure. What contributed to the success of the operation was the use of heparin, the invention of which was one of the mile stones in devel- opment of vascular surgery [ $I]$. Only five years later, in 1951, the technique of endarterectomy in treatment peripheral arterial occlusive disease (PAOD) was used by the American surgeon, E.J. Wylie [2]. In the 1950s and 1960s it was a basic method of surgical treatment used for the treatment of patients with advanced PAOD [3-5]. The method was also successfully performed in the leading hospitals in Poland, the proof of which are the currently cited papers from the 1970s written by the 
surgeons from the Vascular Surgery and Transplantology Clinic of Surgery Institute at the Warsaw Medical Academy in those days run by professor Jan Nielubowicz [6]. After the introduction of vascular prosthesis by de Bakey in 1957 and development of the aorto-femoral bypass grafting technique, endarterectomy gradually lost its meaning. In our paper we would like to remind that with a proper qualification of patients it is still a valuable and effective method of treatment.

There are three main variants of endarterectomy: direct endarterectomy which consists in making an longitudinal incision in the artery at the whole length of the atherosclerotic plaque and removing the plaque under adequate vision; semiclosed endarterectomy, used particularly in treatment of atherosclerosis of external iliac arteries, where the atherosclerotic plaque is removed with the Vollmar and Cannon ring loops pulled between the two incisions in the artery proximally and distally from the atherosclerotic plaque, and the subject of our paper - eversion endarterectomy consisting in removal of the atherosclerotic plaque under adequate vision by eversion of the artery after it has been cut. Among the surgeons whose contribution to the development of eversion endarterectomy was most significant one should mention J.H. Harrison [7] and T. Inhara $[8,9]$.

\section{Material and methods}

The study included nine patients (seven men and two women), at the age of 54-81, operated in the years 20I3-20I4. All these patients suffered from PAOD of the lower limbs, with critical limb ischaemia and advanced atherosclerosis, not qualifying or with contraindications for endovascular treatment (TASC II C and D).

In the group of our patients, one had a femoral-popliteal bypass done in the past, another one underwent an amputation of the right lower limb due to ischaemic necrosis. Some of the patients had atherosclerotic lesions not only in the iliac section (see the chart).

The patients for all the surgeries performed by us were qualified on the basis of the pre-operative CT angiography and/or USG Doppler. All the patients were operated through the retroperitoneal approach.

Out of nine eversion endarterectomies performed by us, seven were elective surgeries and the other two were emergency surgeries as they were done in the patients with acute limb ischaemia.

The lower limb ischaemia advancement was assessed with the use of Rutherford's scale (in the patients qualified for elective surgeries). Five patient had acute ischaemia (R4 and higher); in two patients chronic ischaemia manifested in intermittent claudication (R3).
The advancement of atherosclerotic lesions was assessed on the basis of TASC II. All our patients had type $C$ or D lesions - unilateral or bilateral, significant and long stenosis of the external iliac artery extending into the common femoral artery. In two patients treated as emergency cases, eversion endarterectomy was in fact a method applied during re-surgeries.

In both cases the reason for re-surgery was acute arterial thrombosis causing acute limb ischaemia in the patient operated a few hours before. In both cases thrombosis was caused by limitation of blood supply to the operated vessels by advanced atherosclerosis of the external iliac arteries.

The first of these patients was operated in elective surgery planned to remove a false-aneurysm of the common femoral artery in the anastomosis with the prosthesis (after the femoral-popliteal bypass became blocked). During the primary surgery the aneurysm was removed and the missing section between the external iliac artery and the deep femoral artery was replaced with use of the great saphenous vein. A few hours after the surgery acute limb ischaemia occurred caused by thrombosis of the implanted vein section. CT angiography done before the re-surgery revealed advanced atherosclerosis causing significant limitation of blood flow in the external iliac artery. During the re-surgery the thrombus was removed by eversion endarterectomy.

In the next patient, the first surgery was thrombectomy performed to treat acute ischaemia of the lower limb. The first (incorrect) diagnosis was embolism of the common femoral artery. The thrombus from the common and superficial femoral arteries and from external iliac artery was removed. The inflow from the iliac artery was assessed as sufficient in spite of the clear presence of atherosclerotic lesions (during the use of Fogarty's catheter). Also in this case, a few hours after the surgery acute limb ischaemia occurred - caused by thrombosis of the common femoral artery and external iliac artery. CT angiography revealed advanced long-segment atherosclerosis of the external iliac artery resulting in significant stenosis of this artery. In this case also eversion endarterectomy was performed. In five patients supplementary patch plasty of the common femoral artery was performed; in four other patients this procedure was not applied. One of the patients for whom patch plasty was applied, was treated in emergency surgery (acute ischaemia).

The short-term effectiveness of the applied treatment was assessed on the basis of clinic examination, taking into account the presence of blood flow in the groin or peripheral blood flow in the lower limb, absence of rest pain and improvement of return flow in capillary vessels of the foot. 


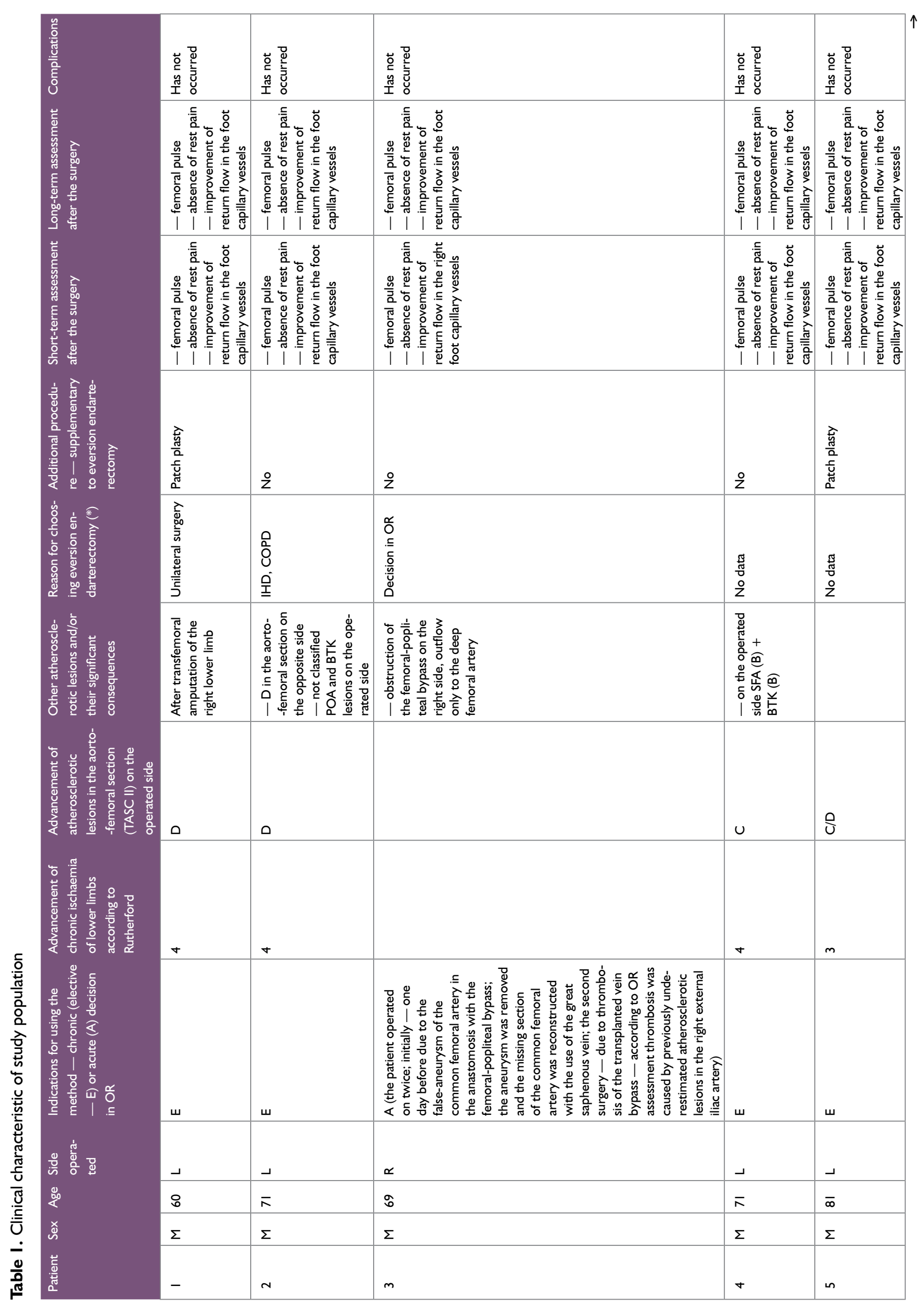




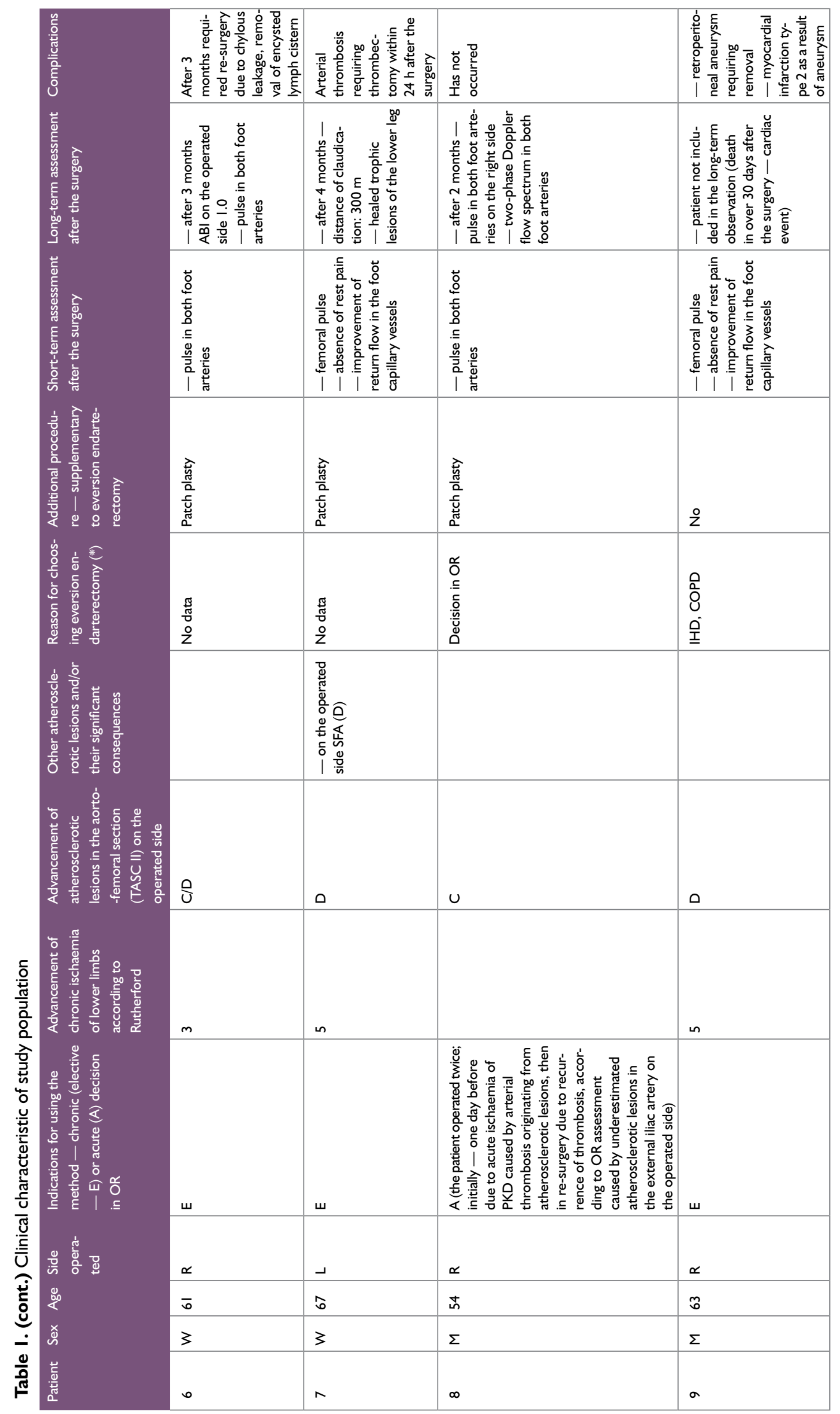


The long-term effectiveness was assessed in a similar way; however, also ABI, a distance of claudication, healing of ischaemic ulcer, as well as the presence and character of arterial blood flow revealed in USG Doppler examination were applied.

In all the cases, eversion endarterectomy of the external iliac artery, consisting in cutting the external iliac artery at the bifurcation of the common iliac artery was performed. By cutting the artery at this level it was also possible to partly remove the plaque from the outlet of the internal iliac artery and the end section of the common iliac artery. Next, the cut artery to the level of the inguinal ligament was everted and the atherosclerotic plaque was removed. Then the cut artery was reconnected end to end. In cases where the plaque extended into the common femoral artery narrowing it substantially, supplementary endarterectomy of the femoral artery and its bifurcation with patch plasty was performed from a separate groin access.

\section{Results}

For all our patients the restoration of patency by eversion endarterectomy was successful; it resulted in improvement of blood flow in the limb and reduction of the patients' ailments. In three patients post-operative complications occurred, all of them in the patients who had elective surgeries. Two complications occurred directly after the surgery. In one case it was thrombosis in the operated iliac artery. The patient was re-operated - a successful thrombectomy was performed - and in further postoperative treatment no complications were observed. In another patient, in the early postoperative period a retroperitoneal aneurysm occurred on the operated side. The patient required blood transfusion and then re-surgery - the aneurysm was decompressed; the site of bleeding was not exposed. The last complication was remote. The patient developed chylous leakage from the postoperative wound. USG showed an encysted lymph cistern corresponding with a lymphocoele. The lymphocoele was removed surgically. No further complications were observed.

In our patients' group we noted one serious adverse extra-vascular event - a myocardial infarction type 2 , which was a complication of the retroperitoneal aneurysm described above.

\section{Discussion}

The eversion endarterectomy technique was first used by Inhara in 1962 and described in 1965 [8]. In this method, eversion of the external iliac artery is done after the common femoral artery has been cut through the groin access. After ligation of the inferior epigastric artery and the deep circumflex iliac artery, the artery is dissected through the retroperitoneal approach at its whole length to the bifurcation of the common iliac artery into the external and internal arteries. The isolated external iliac artery is prepared under the inguinal ligament, moved entirely to the open retroperitoneal space and everted at $2 / 3-3 / 4$ of its length. Then the atherosclerotic plaque is cut off (most frequently the plug occluding the vessel) and the artery is everted back. In the next stage. a longitudinal arteriotomy is done along the distal section of the common iliac artery and the proximal section of the external iliac artery and direct endarterectomy of this section is performed. Then, through the arteriotomy lumen, eversion of the proximal section of the external iliac artery is performed in order to remove the remaining atherosclerotic plaque. Finally, the correct position of the artery is restored', the arteriotomy in the proximal section is closed with a primary suture or using a patch, the distal section is repositioned under the inguinal ligament and reconnected to the femoral artery.

In July 2013, "European Journal of Vascular and Endovascular Surgery" published the article by the German authors - Nagy et al. [10]; they describe the modified technique of endarterectomy of the external iliac artery which they called, to contrast it with the method described by Inhara, antegrade endarterectomy. The authors of this paper perform eversion endarterectomy by cutting the external iliac artery through the retroperitoneal access just below the bifurcation of the common iliac artery. This is the technique we used in the treatment of our patients. The authors of the paper emphasise that, in contradiction to Inhara's method, eversion is done in comfortable circumstances after moving the cut external iliac artery to the groin area under the inguinal ligament without the necessity to ligate the inferior epigastric artery and the deep circumflex iliac artery - significant for collateral circulation.

Endarterectomy is one of the basic techniques used by a vascular surgeon, particularly in the treatment of atherosclerosis of carotid and common iliac arteries. However, the use of this method for other arteries is less common and less known. Such information can even be found in the vascular surgery textbook "Rutherford's Vascular Surgery $8^{\text {th }}$ Edition", where one can read that the currently rare use of endarterectomy is caused, on one side, by good results of bypass grafting achieved in this anatomical segment as well as recognized effectiveness of endovascular treatment, but also by the fact that there is a gradually smaller number of surgeons who feel comfortable using this method due to the lack of experience and lack of possibility to learn this method. It is also raised that it is not a totally "easy" 
method and it carries the risk of substantial blood loss. Frequent presence of the atherosclerotic lesions in the other homonymous artery considerably limits indications for this method. Nevertheless this method has certain undeniable advantages. It allows maintaining the normal physiological and anatomical conditions of the blood flow. It also allows avoiding implantation of foreign material and related complications, including infections, and at the same time not excluding this option of treatment in the future, which in view of a long-term progression of atherosclerotic disease allows extending the period during which other surgical solutions can be applied. In contrary to occasionally used extra-anatomical bypass graft, e.g. femoral-femoral crossover graft or axillo-femoral graft, there is no risk of ischaemia of the other limb. Another advantage of endarterectomy in general, including eversion endarterectomy, is a possibility to maintain better sexual potency, because it allows avoiding preparation of the aortic walls and common iliac arteries. The group of patients who would potentially benefit better from endarterectomy rather than bypass in aortoiliac segment includes the patients with atherosclerotic lesions not extending to common iliac arteries, the male patients who, apart from ischaemia of lower limbs, suffer also from erection disorders and diagnosed atherosclerotic stenosis or occlusion of an internal iliac artery, the patients with atherosclerotic occlusion of external iliac arteries with considerable risk of infections after bypass procedures or for whom, due to their narrow blood vessels, bypass is technically difficult, the patients with "localized" atherosclerotic lesions, and last but not least, the patients with bifurcated graft with end-to-side anastomosis who need to have the prosthesis removed due to infection - in such cases the authors of this paper suggest endarterectomy as one of the alternative "rescue" solutions [10-17]. Currently, the value of direct aortoiliac endarterectomy has been reminded by Connoly and Price [12], and yet, they raise a question in the title of their paper whether aortoiliac endarterectomy is a lost art. Their paper is a retrospective analysis of 200 patients 10 years after surgical treatment of aortoiliac occlusive disease, where 39 patients were treated with endarterectomy and the rest with bifurcated aortofemoral bypass. The authors report that 10 year after the surgery, the primary patency after endarterectomy was $89.2 \%$ and after bypass $-78.0 \%$. Very interesting information regarding endarterectomy can be found in the paper from 2010 by Chiu et al. - a retrospective analysis of various surgical methods of treatment of atherosclerotic aortoiliac disease [13]. In the summary this retrospective analysis informs that the morbidity rate related to endarterectomy is the smallest in comparison with aortofemoral bypass and iliac-femoral bypass - $12.5 \%$,
$16 \%$ and $18.9 \%$, respectively; $(p<0.05)$. Similar results were observed for mortality rate after surgery $-2.7 \%, 4.1 \%$ and $2.7 \%$, respectively; $(\mathrm{p}<0.000 \mathrm{I})$. At the same time, the authors of the analysis did not note any statistically significant differences between all the tree reconstruction methods, which allows for the conclusion that these methods are comparable: 5 -year patency of the treated section was $88.3 \%$ (for endarterectomy), $86.3 \%$ (for aortofemoral bypass) and $85.3 \%$ for iliac-femoral bypass.

Various authors give their own observations of patients after endarterectomy. The primary patency after 5 years is reported at $80-90 \%$, the secondary one at $88-96 \%[10,11,13,15]$. Also long-term observations can be found where cumulative patency 10 years after the surgery reaches even $85-89 \%[12,18]$. An intensive development of endovascular methods of treatment has resulted in constantly improving results of treatment of aortoiliac occlusive disease and increased qualification of patients for transcatheter treatment. The primary patency of such treatment after 4-5 years fluctuates between 77 and $90 \%$, the secondary one after 3-4 years - between 94 and $96 \%$ [18-20]. Nevertheless, not all cases can be treated with endovascular methods. There are numerous reports in scientific literature, both dating nearly 50 years ago $[9,21-24]$ and more contemporary $[10,11,16,17]$, presenting the additional advantages of eversion endarterectomy. The eversion of the artery gives a visual control over endarterectomy, allows for more radical removal of the atherosclerotic plaque from the artery as well as removal of lesions in the arteries which are less accessible for classic endarterectomy. It is possible to remove lesions from long sections of the artery this advantage was used also in restoring the patency in long sections of the superficial femoral artery [25]. The technique also allows avoiding long longitudinal arteriotomy and long sutures that may additionally narrow the artery lumen.

What should be emphasized at this point is the number of advantages of the retroperitoneal approach in comparison with the transperitoneal approach. The retroperitoneal surgical approach to the iliac aorta and arteries aims at reduction of operational injury for the patient. Due to the fact that the peritoneum is not opened, the risk of adhesions (which may result in occlusions in the future) is reduced, the time of postoperative paralytic ileus is shortened, the patients sooner start oral intake of foods and fluids. Also, less pain intensity reported by the patients and smaller consumption of analgesics have been noted. The risk of postoperative pulmonary complications is smaller, patients are extubated sooner and their stay at ICU is shorter [26-29]. 
The fundamental reason why we decided to perform eversion endarterectomy in our patients (not the aorto-femoral bypass grafting) was the fact that as a result we gain the possibility not to leave a vascular prosthesis (which is a foreign object always posing a risk of infection) in a patient's body. All our patients fulfilled the criteria for critical ischaemia and advanced atherosclerotic lesions, did not qualify (or there were contraindications) for endovascular treatment (TASC II C and D), and the location of atherosclerotic plaque in the external iliac artery allowed performing this procedure.

\section{Conclusions}

Eversion endarterectomy of an external iliac artery is a relatively simple surgical method of known and proven effectiveness. In the age of sophisticated endovascular techniques and extensive use of vascular prosthesis, it constitutes a bit forgotten but valuable supplement to an armamentarium of surgical methods in the treatment of atherosclerosis of the aortoiliac segment. With the appropriate qualification of patients this method may still be effectively used in these patients in whom performing endovascular surgery is impossible or contraindicated, or as an alternative for bypasses, especially in cases with a high risk of prosthesis infection.

\section{References}

I. Menard MT, Belkin M (2014) Aortoiliac disease: direct reconstruction. In: Cronenwett JL, Wayne Johnston K (ed) Rutherford's Vascular Surgery 8th Edition. Vol 2. Saunders Company, Philadelphia: I70I-1722.

2. Wylie EJ, Kerr E, Davies O (195I) Experimental and clinical experiences with the use of fascia lata applied as a graft about major arteries after thrombendarterectomy and aneurysomorrhaphy. Surg Gynecol Obstet; 93: 257.

3. Butcher HR Jr, Jaffe BM ( 197I) Treatment of aortoiliac occlusive disease by endarterectomy. Ann Surg; 173: 925-932.

4. Kruse CA, Kirby FG (1957) The surgical treatment of arteriosclerosis occlusive disease (thrombendarterectomy in selected cases). Calif Med; 86: 108-II4.

5. Connolly JE, Stemmer EA (1970) Eversion endarterectomy of the aortoiliofemoral arteries - a three-year experience. Arch Surg; 100: 46I-469.

6. Przetakiewicz Z, Przemaski I (1997) Technika udrożnienia dolnego odcinka aorty i tętnic biodrowych drogą wynicowania. Pol Przegl Chirurg; 10: 927-930.

7. Harrison JH, Jordan WD, Perez AR (1967) Eversion thrombendarterectomy. Surgery; 6I: 26-30.

8. Inahara $T$ (1965) Surgical treatment of aortoiliac atherosclerosis. Am J Surg; 58: 960-968.

9. Inahara T (1972) Endarterectomy for occlusive disease of the aortoiliac and common femoral arteries. Evaluation of results of the eversion technic endarterectomy. Am J Surg; 124: 235-243.
10. Nagy GCs, Wunsch M, Jordan A, Lange K (2013) Antegrade eversion endarterectomy of the external iliac artery. Eur J Vasc Endovasc Surg; 46: 103-109.

II. Vitale GF, Inahara T (1990) Extraperitoneal endarterectomy for iliofemoral occlusive disease. J Vasc Surg; I2: 409-4I3.

12. Connolly JE, Price T (2006) Aortoiliac endarterectomy: a lost art? Ann Vasc Surg; 20: 56-62.

13. Chiu KWH, Davies RSM, Nightingale PG, Bradbury AW, Adam DJ (2010) Review of direct anatomical open surgical management of atherosclerotic aortoiliac disease. Eur J Vasc Endovasc Surg; 39: 460-47I.

14. 14. Metcalfe MJ, Natarajan R, Selvakumar S (2008) Use of extraperitoneal iliac artery endarterectomy in the endovascular era. Vascular; 16: 310-315.

15. Radoux JM, Maiza D, Coffin O (200I) Long-term outcome of 121 iliofemoral endarterectomy procedures. Ann Vasc Surg; 15: $163-170$.

16. Donohoe CL, Dowdall JF, McDonnell CO, O'Malley MK, O'Donohoe MK (20II) Eversion endarterectomy for external iliac artery occlusive disease. Vasc Endovascular Surg; 45: 45-50.

17. Sirangulo C Jr, Langer B, Kauffman P, Bouabci AS, Aguiar ET, Cinelli M Jr (1991) Arterial reconstruction of the iliofemoral segment by eversion endarterectomy. Rev Hosp Clin Fac Med (Sao Paulo); 46: 63-73.

18. Balzer JO, Thalhammer A, Khan V, Zangos S, VogI TJ, Lehnert T (2010) Angioplasty of the pelvic and femoral arteries in PAOD: results and review of the literature. Eur J Radiol; 75: 48-56.

19. Yuan L, Bao J, Zhao Z, Feng X, Lu Q, Jing Z (2014) Endovascular therapy for long-segment atherosclerotic aortoiliac occlusion. J Vasc Surg; 59: 663-668.

20. De Roeck A, Hendriks JM, Delrue F et al (2006) Long-term results of primary stenting for long and complex iliac artery occlusions. Acta Chir Belg; 106: 187-192.

21. Inahara T (1975) Evaluation of endarterectomy for aortoiliac and aortoiliofemoral occlusive disease. Arch Surg; I I0: 1458-1464.

22. Connolly JE, Stemmer EA (1972) Simplified technique of eversion endarterectomy for aortoiliofemoral occlusive disease. Arch Surg; 105: 520-523.

23. Harjola PT, Tala P (1970) Eversion endarterectomy in aortoiliofemoral arteriosclerotic obstructions. Scand J Thorac Cardiovasc Surg; 4: 65-68.

24. Inahara $T$ (1979) Eversion endarterectomy for aortoiliofemoral occlusive disease - a 16 year experience. Am J Surg; 138: 196-204.

25. Treadwell T, Korompai MD, Hayward RH (1977) Eversion endarterectomy: a new application of an old technique. Cardiovacs Dis; 4: 303-310.

26. Kalko Y, Ugurlucan M, Basaran M et al (2008) Comparison of transperitoneal and retroperitoneal approaches in abdominal aortic surgery. Acta Chir Belg; 108: 557-562.

27. Arko FR, Bohannon WT, Mettauer M et al (200I) Retroperitoneal approach for aortic surgery: is it worth it? Cardiovasc Surg; 9: 20-26.

28. Sicard GA, Reilly JM, Rubin BG et al (1995) Transabdominal versus retroperitoneal incision for abdominal aortic surgery: report of a prospective randomized trial. J Vasc Surg; 21: 174-181, discussion 181-183.

29. Djedovic M, Rustempasic N, Djedovic S et al (2013) Comparison of transperitoneal and retroperitoneal approach in aorto-iliac occlusive disease. Med Arch; 67: 249-25I. 